\title{
Architectures and Interfaces for a Micro Factory Concept
}

\author{
Niko Siltala, Riku Heikkilä, Asser Vuola, and Reijo Tuokko \\ Tampere University of Technology, Department of Production Engineering \\ Korkeakoulunkatu 6, 33101 Tampere, Finland \\ \{niko.siltala, riku.heikkila, asser.vuola, reijo.tuokko\} @tut.fi \\ http://www.tut.fi/tte
}

\begin{abstract}
So far the desktop manufacturing is mainly done as islands of process modules or in some seldom cases the desktop factory is created in form of manufacturing line. Tampere University of Technology has been working on such desktop factory concepts for years and come out a microFactory concept (TUT- $\mu \mathrm{F})$. The paper discusses architectural aspects and proposes some solutions for them. It specifies also two main mechatronic interfaces used for such modular desktop factories - 1) the cell to cell interface and 2) cell internal process module interface. Main parts of the specifications are represented. These can be utilised for building the desktop production line from easily integrated modules.
\end{abstract}

Keywords: desktop factory, micro factory, module interfaces.

\section{Introduction}

This paper will present the recent development and documentation of the architectures and interfaces for a micro factory concept developed at Tampere University of Technology (TUT). The concept is called TUT-Microfactory® shortened here as TUT- $\mu$ F. The concept has long roots throughout the series of national projects starting in 2000 from project TOMI (Towards Mini and Micro Assembly Factories). Since then the concept has gone through several evolutions and grown more mature as a concept. $[1,2]$

The different mini and micro factory concepts have been presented since early $90 \mathrm{~s}$ [3]. However, the research is typically focusing on single machines and not that much on integration of stand-alone processes and machines into larger manufacturing entities like production lines with integrated material logistics. Motivation to research and development of micro and desktop manufacturing and factories leans on technological, business and sustainability reasons. The first because there exist a need for manufacturing and assembly of high-precision, miniature products; and new innovations and technical solutions offer today smaller sized components for building the systems. The second, because there exist a need to postpone the product customisation as late as possible and closer to the customer; and possibility to utilise new business models. The latter because arrival of new requirements for space savings, energy consumption and utilisation of resources on factory floor. Modular systems built from reconfigurable and re-usable modules support as well the sustainability aspect. 
Opening the architectures and interfaces the TUT- $\mu \mathrm{F}$ concept will be available for others to join and utilise the concept and also to provide the new compatible process modules. System integration will become easier as the shared interfaces (both mechatronic and communication) are used between modules from different origins.

\section{Method}

In many cases the mini, micro or desktop factory concept or application is working like an isolated island, which is served by a human operator and assisting him or her by performing tasks which are difficult for a human. Integration of the material flow between different stations or integration on the control side is the typically missing. The new option for micro factories is an integrated assembly or manufacturing system where workstations or cells are aggregated into a line or even bigger factory system. The cells itself can also be aggregated from modules with standardised interfaces. The proposed TUT- $\mu \mathrm{F}$ concept is based on the latter integrated solution having as one objective to provide an architecture where the process specific modules can be composed freely into different layouts with minimum limitations; modularity, reconfiguration and re-use are well presented; each module is self-contained with their controls; etc.

\section{Architectures}

\subsection{Cell}

The outer dimensions of the base module (i.a. cell frame) used in the TUT- $\mu \mathrm{F}$ are 200 x 300 x $230 \mathrm{~mm}$ (Width x Depth x Height). The cell size is extendable in height as the process modules can be stacked on top of the base module. The depth of the base module is allowed to vary within the given range depending on the need of cell manufacturer and implemented processes. The line width is the most rigid dimension off the base module. The inside work space is 180 × 180 x $180 \mathrm{~mm}$. This offers small modules easily movable by human means, but still sufficient workspace volume needed for assembling typical handheld electronic products and other mini or microsized products. Cell communication architecture is presented in [6].

The base module can have other supplementary features implemented like the clean room or temperature controlled environment, frequency (wavelength) blockage shielding e.g. in case of laser processes.

The cell itself and the processes it is capable to perform are specified with the Emplacement concept and Blue Print files [5]. This is offering a novel method for electronically describe the features and capabilities of a module. This information can be utilised during the system design and use [4].

Cell Internal Architecture. One main cell internal interface is the process module interface on top of the cell, which can be repeated allowing a stacking structure. The electrical supply and communication through ethernet is available. Modules are intended to be self-contained and independent modules with their own controls. The base plate of the base module can be used to connect transport and process related sub-modules. The matrix of attachment locations is provided through thread holes. 


\subsection{Line Layout}

The base modules can be relatively freely and easily set up on different line layouts. The layout can be line with branches, even some loops are allowed. The Fig. 1 illustrates one possible layout example including a carrier based transport system.

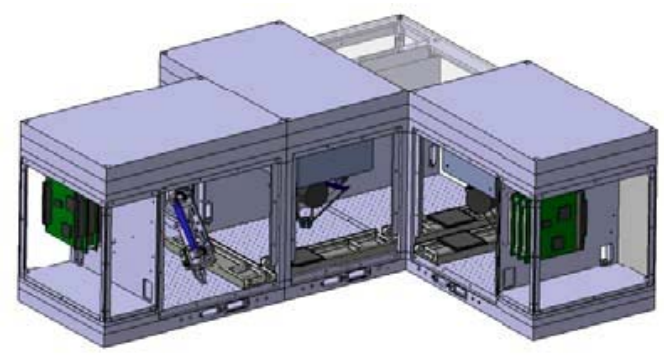

Fig. 1. Example of TUT-Microfactory layout [1]. Represented are four cells with different manipulators and conveyor system connected together.

Even the maximum freedom for combining cells together is sought, some rules still apply. The Fig. 2 illustrates some of these rules. The arrow inside the cell points the supposed flow direction of the main product (from left to right). The male and female connectors are differentiated by the shape and correspondingly the purpose of the connection with colours. The basic rule is that male and female connectors with the same colour can be connected together. As one can recognise, the mechanical connection is possible in some other configurations, but the purpose of connectors will then mismatch which leads to a critical signal failure. However, some techniques could be used in order to get such kind of layouts accepted e.g. by using a cross-wiring adapter or change-over switch for signals.
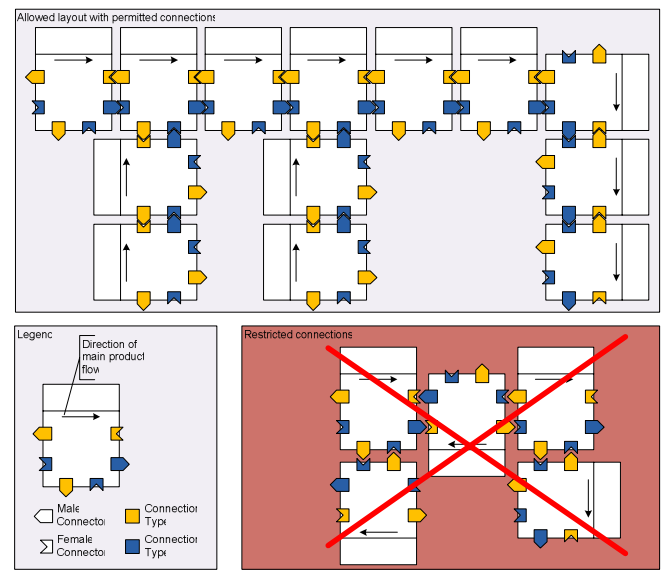

Fig. 2. Allowed and restricted cell connections within the TUT- $\mu \mathrm{F}$ architecture 


\subsection{Transport}

Three different forms of transport are proposed: pallet based, product on conveyor and product on air. The first is traditional pallet based approach using carriers to transport the product and other materials between workstations. Carrier can be based on e.g. DIN 32561 [7]. Simple belt conveyors or other means can be used for making the transport. The advantages are stable support for differently shaped and sized parts travelling through the system. Drawbacks are the product specific caves or other mountings made to pallet and returning of the pallets back to the beginning of the system. The carrier based system within the TUT- $\mu$ F concept is discussed in [3].

In the second approach the product is transported without a specific carrier from a workstation to next on a flat belt conveyor. This approach is suitable for the first case so it can also move the pallets. Here the advantage is the universality of the solution. There is no need for product specific fixtures on the pallets. However the main drawback is the additional requirements for the product. It needs to have size large enough, flat bottom maintaining the product in stable position during the transport between the workstations and manipulations, and surface quality for the product should not be very high (as the pallet is not protecting the product). In many cases it is required to have a separate fixture inside the cell for supporting the product during the assembly.

In the third approach the product is passed on air with the help of manipulators between the parallel workstations. This would require means to grip the part and transfer it between the processing places. The manipulator making the assembly can be utilised for the transfers, if the range is large enough.

In all cases the by-pass of the cell must exist. In cases when the workstation gets broken and production needs to continue or there are multiple stations performing the same process task, the by-pass gets mandatory. The performance of the overall system depends on the way the by-pass is implemented.

\subsection{Controls and Communication}

Ref. [3] discusses the possible control approach followed by the concept. One instance of the cell internal control and communication architecture is opened in [6].

The communication between modules is done over standard ethernet protocols like TCP/IP and UDP/IP (max. 100Mbit). Each base module has an embedded switch distributing the connection to neighbouring cells as well to the control modules composing the cell.

\subsection{Integration of Other Modules}

The TUT- $\mu \mathrm{F}$ approach and architecture needs to be easily integrated with other kind of systems like lines or cells with larger size (macro production) or with different architecture. These cases adapters connecting two architectures together are used.

\section{Results}

\subsection{Interfaces}

The standardised interfaces are the main offering of the proposed concept even they are yet only "company specific standards". These are introduced and discussed in details on the following chapters. 
Cell-to-Cell. The cell to cell interface for TUT- $\mu \mathrm{F}$ is specified in [9]. The main part of the interface is represented in the Fig. 3, which shows the dimensional drawing of the interface. It shows the details of the mechatronic interface including the intended features, dimensions, connectors used. In addition to mechanical information the electrical signals, pin assignments, communication channels, origin, etc. are specified in the document.

The features available in the specification are: Opening for the product to pass through. At minimum 180 x $180 \mathrm{~mm}$ opening shall be available. In the practice the application may use smaller area; Fastening and fixation (1A \& $1 \mathrm{G}$ in Fig. 3). Hinges and plates can be used to fixating cells together; Positioning function (1B \& 1F). The pins and holes or bushes are used to accurately locate the mating interfaces. The pin (1F) serves as the origin of the interface.; Electrical connection and communication (1C \& 1E). The electrical supply of $24 \mathrm{VDC}$ is supplied through the larger pins. Ethernet (max.100Mbit) and handshake signalling for the product exchange between cells are using the smaller signal pins; Pneumatics distribution line in (1D).

The connectors are on the same base line ([A] in Fig. 3), from which the cell floor at processing area is located $20 \mathrm{~mm}$ above and cell bottom $10 \mathrm{~mm}$ below. The cell floor is allowed freely to go below the defined, have openings for manufacturing processes, etc.

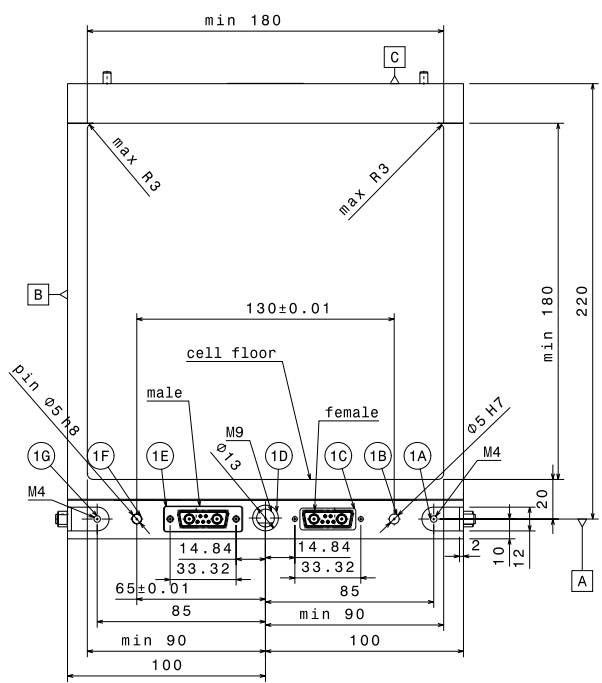

Fig. 3. Cell-to-cell interface. View at front.

Transport. Transport interface for the main product flow is specified in [9]. There are specified three different options for cell to cell transport of products on the main production flow. Two of them are conveyor based approaches and third is through air i.e. manipulator is taking care of the transport over the cell boundary.

The conveyor based approaches define where the conveyor shall be located, how the used conveyor width is designated. The main principles are that in the option A 
the conveyor is located back half of the workspace leaving the front part for assembly and feeding actions. In the option B the conveyor is located at the centre line of the side interface of the cell i.e. going through at the centre of the workspace of the cell from the left to right.

Process Module. The process module interface for TUT- $\mu \mathrm{F}$ is specified in [10]. The main part of the interface is represented in the Fig. 4. It shows the dimensional drawing of the interface with the details of the mechatronic interface including the intended features, dimensions and connectors used.

The interface specification [10] defines the features like: Minimum available opening for the process modules above is defined $(180 \times 180 \mathrm{~mm})$; Orientation feature with the use of pins (2B) in Fig. 4, which defines the orientation of the interface; Interface origin is located concentric with the left side pin of (2B); Fastening of the process module with bolts having M4 thread; Electrical supply and communication. The connection contains power for 24VDC and ethernet communication. Additional communication channels can be passed through parallel with ethernet. As option are defined pin assignments for busses like USB, industrial fieldbuses, etc. The specification document defines the connectors, electrical signals, pin assignments, etc.

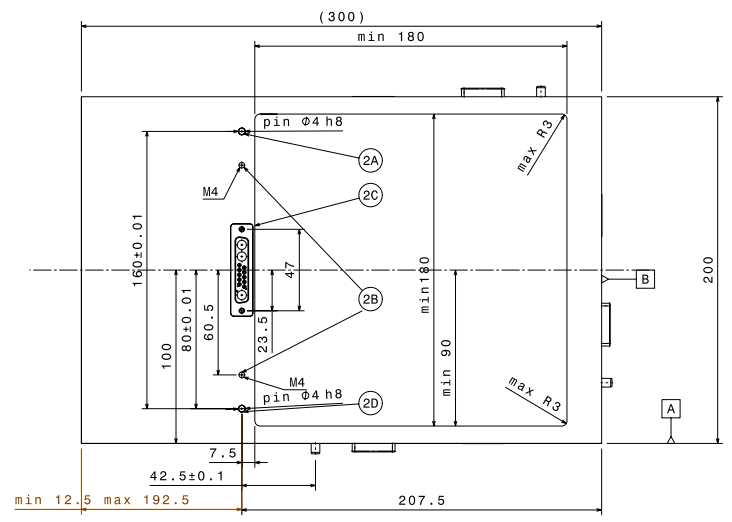

Fig. 4. Process module interface. Top view.

The process modules can be stacked one top of each other. This will be very useful in cases e.g. when on top of the manipulator module there is a vision module. The specification defines the base unit for the module height, from which the real implemented module is integer multiplication. The consumed height of the module will be included into the module designation information. If stacking is not allowed for some reason it is also described by the designation.

Manipulator - End effector. TUT- $\mu$ F concept utilises the ISO 29262 [8] for the interface between the manipulator and end effector.

Feeding. Ref. [3] discusses some feeding methods utilised in TUT- $\mu$ F like tray and flexible feeding. The interface specification for the feeding is not yet stabilised and found its latest form within our concept for single feeders. One of proposed alternative 
is to use Fuji IP/QF series feeder interface. However, it will be sure that several different options will be finally available within the concept, as one single approach cannot be serving all different objectives like mechanical scalability of size (both component and feeder), feeding methods, etc.

In case the feeder is independent and self-contained module, it can be even connected through the cell interface (See Fig. 3)

\subsection{Standardisation}

The objective is extensive use of the existing standards. However as we are breaking new areas no standards yet exist. The field of micro factories is relatively new and this is one of the first attempts to standardising the interfaces used for such environments.

The paper mentions some standards, which are applied within the concept like [7, 8]. It also presents some specifications which are describing the interfaces used in the TUT- $\mu \mathrm{F}$ concept. These specifications are currently on the development phase and TUT will publish them through some channel later this year. One possible channel could be Evolvable Assembly Systems Environment (http://www.eas-env.org/).

\section{Conclusions and Future}

The paper presents the details of some key interfaces for the TUT-Microfactory (TUT- $\mu \mathrm{F}$ ) concept, which are further developed and documented within our currently ongoing Mz-DTF project at Tampere University of Technology. By publishing the interfaces we invite others to join the development of the specifications and to utilise these in order to be able to realize multi-vendor micro and desktop factory systems based on components and modules compatible with each other as they all basis on the same shared definitions and architecture.

In future we are planning to extend the existing interfaces and adding new ones to the set of specifications in the TUT- $\mu \mathrm{F}$ concept. The solidification of our specifications through real standardisation will be also included in the future work.

\section{References}

1. Heikkilä, R.H., Karjalainen, I.T., Uusitalo, J.J., Vuola, A.S., Tuokko, R.O.: Possibilities of a Microfactory in the Assembly of Small Parts and Products - First Results of the M4project. In: Proceedings of IEEE International Symposium on Assembly and Manufacturing (ISAM) 2007, Ann Arbor, Michigan, USA, July 22-25, pp. 166-171 (2007)

2. Heikkilä, R., Huttunen, A., Vuola, A., Tuokko, R.: A Microfactory Concept for LaserAssisted Manufacturing of Personalized Implants. In: Proceedings of 6th International Workshop on Microfactories (IWMF 2008), Northwestern University, Evanston, Illinois, USA, October 5-7, pp. 77-80 (2008)

3. Jarvenpää, E., Heikkilä, R., Tuokko, R.: Logistic and Control Aspects for Flexible and Reactive Micro and Desktop Assembly at the Factory Level. In: Proceedings of 2009 IEEE International Symposium on Assembly and Manufacturing (IEEE ISAM 2009), Suwon, Korea, November 17-20, pp. 171-176 (2009) 
4. Siltala, N., Tuokko, R.: Use of Electronic Module Descriptions for Modular and Reconfigurable Assembly Systems. In: Proceedings of 2009 IEEE International Symposium on Assembly and Manufacturing (IEEE ISAM 2009), Suwon, Korea, November 17-20, pp. 214-219 (2009)

5. Siltala, N., Tuokko, R.: Emplacement and Blue Print - Electronic Module Description supporting Evolvable Assembly Systems Design, Deployment and Execution. In: 6th International Conference on Digital Enterprise Technology (DET 2009), Hong Kong, December 14-16 (in press, 2009)

6. Vuola, A., Heikkilä, R., Prusi, T., Remes, M., Rokka, P., Siltala, N., Tuokko, R.: Miniaturization of Flexible Screwing Cell. In: Ratchev, S., Hauschild, M., (eds.): IPAS 2010, IFIP AICT 315, pp. 309-316 (2010)

7. DIN 32561: Production equipment for microsystems - Tray - Dimensions and tolerances. DIN standard (2003)

8. ISO/DIS 29262: Production equipment for micro-systems - Interface between endeffector and handling system. ISO Draft international standard (2009) (Supersedes DIN 32565 E) (February 2005)

9. TUT- $\mu \mathrm{F}$ : std-0001: Cell Interface. Draft specification (2010) (unpublished)

10. TUT- $\mu$ F: std-0002: Process Module Interface. Draft specification(2010) (unpublished) 